\title{
Design of thermally deformable laminates using machine learning
}

\author{
A. Abdel-Rahman \\ MIT, Cambridge, USA \\ M. Kosicki \\ Foster+Partners, London, UK \\ P. Michalatos \\ Autodesk, Boston, USA \\ M. Tsigkari \\ Foster+Partners, London, UK
}

\begin{abstract}
Recent advances in material science and manufacturing have enabled designers to create objects which respond to changing environmental conditions by controlled deformation, without external mechanical or electrical actuation. The design of such smart materials has mostly been done through trial and error due to their complex nonlinear behavior. This paper will present how this problem is addressed by introducing a novel inverse design workflow. In this, a desired structural deformation is used as an input to a machine learned model which subsequently outputs the required geometric and material properties that will produce said deformation when exposed to an external stimulus. This workflow uses a Generative Adversarial Neural Network (GANN) trained on pairs of input cut-out patterns of laminate layers and their nonlinear Finite Element Analysis (FEA) simulation results. The method offers a significant performance speed-up while maintaining acceptable levels of accuracy, especially at the early design stage. This methodology could be further extended to the design of any nonlinear mechanical deformation.
\end{abstract}

\section{INTRODUCTION}

\subsection{Motivation}

The ability to manufacture components which can change their shape in response to external environmental conditions, is a desire shared across many disciplines ranging from aerospace, medicine, electronics, soft robotics (Majidi 2014) (Wang et al. 2017) to architecture and the building industry (Addington \& Schodek, 2005) (Szokolay 2008).

In architecture, a facade is a part of a building which exchanges the most energy with the external environment. Contemporary buildings, especially those with fully glazed envelopes, must continuously adapt to ever-changing external conditions to protect their interior spaces. This raises the need for precise control of lighting, humidity and solar radiation, with the facade being most often used as an interface. This issue has been traditionally mitigated by static or dynamic shading systems. Such facade components rely primarily on mechanical hinges, sensors, multiple actuation devices and controls. Today most adaptive facades, such as the shading system designed by Jean Nouvel for the south façade of the Arab World Institute in Paris
(1987) or Al Bahr's modular screen in Abu Dhabi by Aedas (2012), are still based on mechanical, usually electrically-driven systems (Kolarevic and Malkawi 2005).

Achieving similar performance should be made possible without the need of mechanically actuated systems, but rather dynamic materials which can react like living organisms. One such example is electrochromic glass, which controls its transparency (and therefore solar gains), by passing electrical current through a layer of coating (Carmody et al. 2004). One of the pioneering examples of a passive and autonomous skin system was developed by Decker and Yeadon (2010) in their homeostatic facade. They used dielectric elastomer attached to silver electrodes on both sides of the façade and wrapped over a flexible polymer core. The silver reflects and diffuses light, while distributing an electrical charge across the elastomer causing the flexible core to bend. This biological approach to dynamic architectural systems remains rare due to many technical challenges and significant prototyping costs of highly customizable components. Therefore, this research is a part of a bigger effort to develop an integrated workflow for the design and 
fabrication of passively actuated, adaptive building facade elements.

\subsection{Passively actuated systems}

One of the most successful approaches to the aforementioned problem, relies on using self-folding kirigami and origami structures or auxetic materials (materials with a negative Poisson's ratio) and transforming various forms of energy into mechanical actuation (Tolley et al. 2014) (Javid et al. 2015) (Shan et al. 2015) (Liu and Bertoldi 2015) (Mousanezhad et al. 2015) (Liu et al. 2016) (Connolly et al. 2016) (Deng et al. 2017) (Rafsanjani and Bertoldi 2017) (Boatti et al. 2017) (Deng et al. 2017). This origamiinspired approach, as shown by Peraza-Hernandez et al. (2014), is challenging when it comes to rapid and precise fabrication of highly complex 3D structures. Most of the currently used folding logic could be divided into two groups: hinge type and bending type. In the hinge type approach, the folds are clearly restricted to structurally pre-determined hinge locations. The bending category on the other hand, does not rely on discrete hinge systems but is based on direct sheet bending triggered by actuation of an active material. This type of actuation allows much greater foldability than using hinges. This occurs because, unless mechanically restricted, the fold can arise at any place and direction to which the actuating force is applied. Bending systems can be manufactured as single or multi-layer laminates containing various distributions and cut out patterns of active and passive material. The laminates are predominantly manufactured as 2D structures, so they could be quickly and accurately fabricated using either lithographic techniques (Zhang et al. 2015, Maune et al. 2010) or additive multi-material 3D printing (Khoo et al. 2015). Boatti et al. (2017) demonstrated that by combining physical experiments and digital simulations, a wide range of origami metamaterials with varying thermal expansion coefficients could be designed. The widespread approach to designing 3D morphologies for such systems is by creating a digital prototype of a $2 \mathrm{D}$ predecessor, with a predefined geometry and/or a pattern of cuts. Then external forces are applied by running a non-linear Finite Element Analysis (FEA) simulation while observing the resulting deformation. Given the complex nature of the simulation, the process requires a sophisticated and highly-accurate simulation software. The FEA simulations are predominately used one way, from a $2 \mathrm{D}$ prototype to a $3 \mathrm{D}$ buckled model. Therefore, designing a geometry and material distribution for a $2 \mathrm{D}$ predecessor becomes a core problem, which has not been well addressed due to its computational complexity deriving by the size of its design space. Xue et al. (2017) tackled it with topology optimization solved with a Genetic Algorithm. This approach, however successful, required a lot of processing time and did not give real-time feedback and design flexibility which are crucial at early design stages. The current methods are time-consuming and difficult to apply, especially when a specific final 3D complex shape is desired. As previously shown, 3D deployable structures are mostly designed by trial and error and no systematic real-time tool exists that could produce an initial multilayer material cut-out pattern for a desired 3D complex shape. Current methods are also not suitable for the early design stage when many topologically different design alternatives should be taken into consideration and where fast feedback is much more valuable than accuracy.

Therefore, implementing a system capable of predicting a material distribution with a controllable level of accuracy, for a given 3D morphology could be of a great benefit to the design process. Recent advancements in the fields of Machine Learning (ML) and cognitive computing (Jordan and Mitchell 2015) provide us with effective methods of building predictive models purely based on data. In the context of designing a 3D morphology the FEA simulations could be providers of well-structured and organized data repositories. Ramprasad et al. (2017) point out that learning from such data repositories in the context of material science can lead to recognition of previously unknown correlations between properties or qualitative and quantitative rules. These can be culminated in surrogate models which could then be used to predict material properties orders of magnitude faster and cheaper and with reduced human effort. The accuracy of such models is adaptive, meaning that as more data accumulates, their power increases. To take advantage of machine learning the data must be structured as input-output pairs. In the context of the discussed problem the input is a desired 3D morphology and the output is a $2 \mathrm{D}$ material cutout pattern which can produce said 3D morphology under a given stimulus.

\section{METHODOLOGY}

In this section a system capable of generating rapid predictions of $2 \mathrm{D}$ cutout patterns from a given $3 \mathrm{D}$ deformation was proposed and tested. The predictions were generated by a generative adversarial neural network (GANN) trained on a data generated by a nonlinear FEA simulation of flat laminates exposed to heat buckle. The laminates were modelled as consisting of layers differing in thermal expansion coefficients. Two geometrical models were created and evaluated. The first focused on 1D deformation of long laminated strips and the second, on a square-shaped laminate exhibiting shell-like behavior. Both models targeted self-folding behavior by using a three-layer laminate structure with two active layers and one passive layer. In such arrangements the passive layer generates negligible mechanical work compared to the active layers when exposed to external thermal stress. When such thermal stress is applied, the active layer is forced to deform, usually axially, while the passive layer is not. This difference in expansion or contraction between the two layers generates localized bending of laminates (Peraza-Hernandez et al. 2014).

The use of Machine Learning (ML) aimed at predicting a $2 \mathrm{D}$ cutout pattern by providing a target 


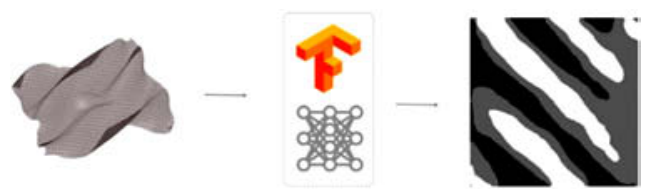

Figure 1. Proposed Workflow. From user-defined deformation through a trained neural network to a predicted material cutout pattern.

deformation. More broadly the task was to map an input data into corresponding output data, which is known in ML literature as supervised learning (Bishop. 2008). Over the past years many algorithms capable of doing supervised learning have been developed, including linear regression, support vector machines, decision trees and deep neural networks. Deep Neural Networks (DNN) have the most widespread use due to their optimal performance over other techniques. One of the most effective solutions using a variation of DNN is pix2pix, a Conditional Adversarial Neural Network (GANN) developed by Isola et al. (2016). The network proved to be effective in a variety of image related tasks e.g. synthesizing photos from label maps, reconstructing objects form edges or colorizing images without the need of hand-tuning the network's architecture or tweaking its parameters. The research presented in this paper adapted a Tensorflow (Abadi 2016) implementation of the pix2pix network trained on pairs of images (Figure 1).

Training data for the neural network was produced by two parametric models capable of generating variations of each type of analyzed geometry. It was developed in Rhinoceros (Robert McNeel \& Associates) $\mathrm{CAD}$ environment using its parametric modelling platform Grasshopper. Each parametric model described a design space of material distribution for a given type of laminate. The design space was then automatically sampled to build a training set for the machine learning algorithm. The detailed description of each model is provided in the next section. The ML algorithm required inputs as set of images with a constant number of pixels. Therefore, a quad mesh, with a constant number of faces, was used to model each laminate. The faces were mapped to pixels and the material properties of each face were encoded in grayscale as follows: the passive layer being white, the active upper layer black and the active bottom layer grey (Figure 2). After running the FEM simulation, the resulting 3D deformation of each sample was also encoded as a displacement map; an image with the same resolution as the matching input image. For the displacement map, the full RGB spectrum was used to maximize discrete displacement states stored for each sample. This approach ensured a clear data structure where each material distribution image corresponded to a displacement image.

All samples were evaluated using Autodesk Explicit, a nonlinear FEA solver to simulate responses of the geometries under thermal stresses.
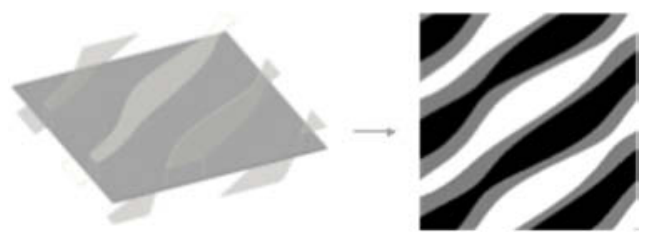

Figure 2. Laminated pieces bitmap representation.

A distributed computing system was used to speedup the simulations. The simulations were parallelized using bespoke software and distributed over 400 CPUs using Foster + Partners on-premise computing infrastructure.

\section{RESULTS AND DISCUSSION}

\subsection{Introduction}

In this section, detailed information of the two models will be presented to demonstrate the effectiveness of using ML in the design of thermally actuated geometries.

\subsection{Model 1}

The first is a simplified model of a family of longitudinal laminate strips $(20 \mathrm{~cm}$ by $6 \mathrm{~cm})$ to study the relative deformation in two dimensions. The strips were meshed uniformly in low resolution (16 by 5 faces) and the left column of vertices was assumed to have fixed nodes. To generate the training and test sets, a total 600 random samples were generated. In each sample, each column of mesh faces was randomly assigned one of three material setting: passive material, passive with an active top layer, passive with an active bottom layer. These iterations were bulk simulated using Autodesk's Explicit Solver according to the following settings. A uniform increase in temperature $\left(15\right.$ degrees $\left.{ }^{\circ} \mathrm{C}\right)$ throughout 1.0 second was introduced to actuate the membrane. To speed up the simulations, and since the tests were not concluded against real physical models, dummy material settings were used to amplify the mechanical deformation. Both passive and active materials had a Young's modulus of $69 \mathrm{MPal}$, Poisson ratio of 0.334 , density of $2700 \mathrm{~kg} / \mathrm{mm}^{3}$ and thickness of $0.01 \mathrm{~mm}$. The first material (active) had a high expansion coefficient of $0.01 \mathrm{~mm} /{ }^{\circ} \mathrm{C}$, and the second material (passive) had a low expansion coefficient: $0.00001 \mathrm{~mm} /{ }^{\circ} \mathrm{C}$.

The next step was the translation of the input models and FEA findings into 2D images for them to be trained using the proposed method. The meshes were mapped into input grayscale bitmaps ( 256 by $256 \mathrm{px}$ ) where grey, white and black expressed the different material settings (Figure 3).

As for the FEA results, the mesh deformation achieved after $1.0 \mathrm{~s}$ was translated into output grayscale bitmaps according to the following strategy: a surface was first reconstructed from the deformed 


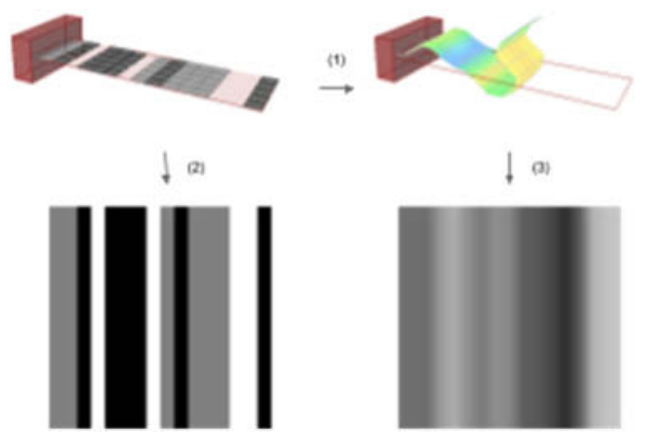

Figure 3. Translation of material settings and deformation to bitmaps (model 1).
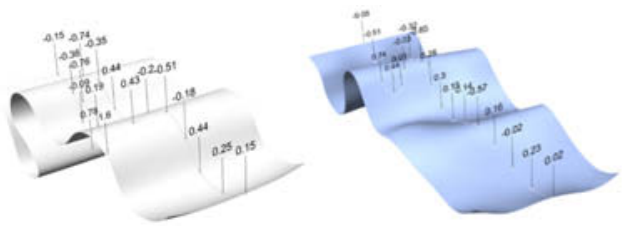

Figure 4. Mean curvature calculation.

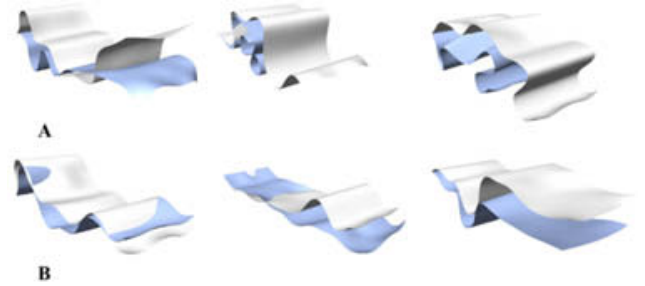

Figure 5. Desired Deformation (white) vs Predicted Defor-mation (blue) A) Samples Performing Poorly, B) Top Perform-ing Samples.

vertices, and since the membranes are treated as linear strips and only the 2D deformation is of interest, the mean curvature was calculated at the middle of the strips in a number of points and mapped smoothly into an output bitmap (from -1 to 1, to 0-255) (Figure 34). The next step was to use the test set output images (desired deformation) and the trained machine learning model to predict input cut out patterns. Lastly, to evaluate the prediction, the predicted cut out patterns were re-simulated using the FEA solver and compared to initial desired deformations.

Figure 5 shows examples of the top performing samples and some that had low performance using the root mean squared error (RMSE). The ones that had large desired deformation had higher error that the flatter examples, however the predicted model was able to capture the general curvature of the desired deformation. The average root mean squared error for all samples is 0.37 (Figure 5-6).

\subsection{Model 2}

Model 2 tested the applicability of the proposed method for more complex doubly-curved deformed

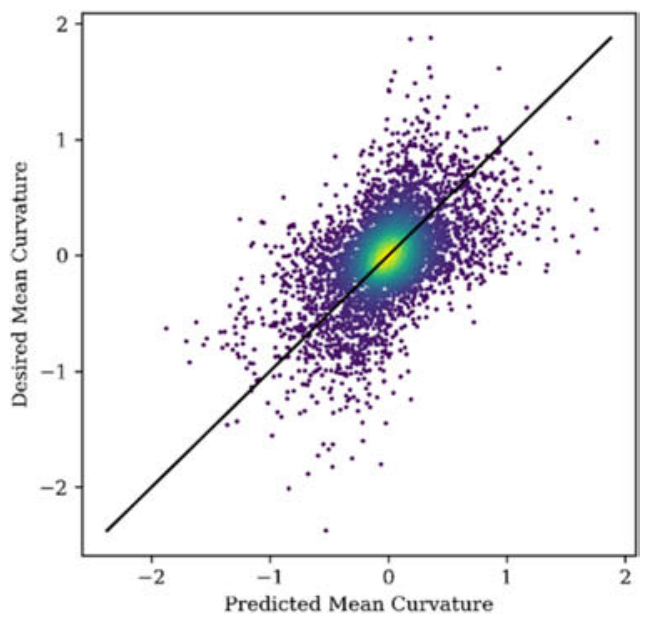

Figure 6. Predicted vs Desired Mean Curvature for all the points in all the samples for Model 1.

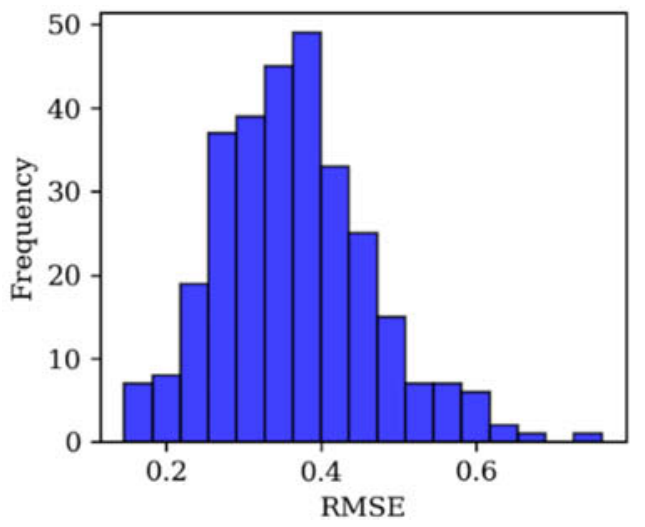

Figure 7. Probability Density Function for Root Mean Squared Error for Model 1.

membranes. The meshes tested in this model were much coarser (41 by 41) and supported at the center of the mesh by a fixed point. To generate a family of random complex cutout patterns, the interference of four random set of wave functions was used to generate 300 3D surfaces (Equation 1).

$$
\begin{gathered}
z=\sum_{i=1}^{4} \quad \cos \left(a *\left(u_{i} * x+v_{i} * y\right)\right)+i * \sin (a * \\
\left.\left(u_{i} * x+v_{i} * y\right)\right)
\end{gathered}
$$

Equation 1: $\mathrm{x}, \mathrm{y}$ and $\mathrm{z}$ are the vertices positions, $\mathrm{a}=1$, $\mathrm{u}$ and $\mathrm{v}$ are lists of four random values from -1 to 0 .

The surfaces generated were then intersected with two planes with different heights to threshold and generate the cutout patterns of the membrane with the three materials settings (Figure 8). These surfaces where consequently batch simulated using the FEA solver with the same material, time and temperature settings as the first model. The cutout patterns were mapped into grayscale input images in the same process as the first example. The simulation results were however mapped into RGB bitmaps where the $\mathrm{x}, \mathrm{y}, \mathrm{z}$ 


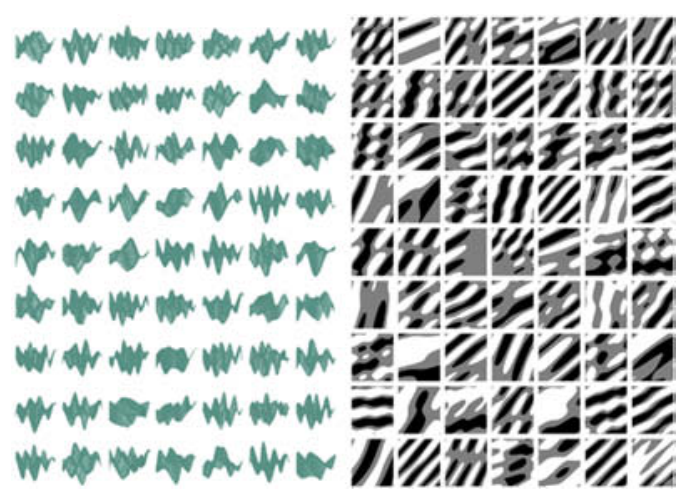

Figure 8. The Random 3D Waves and their Mapping into Cutout Patterns.
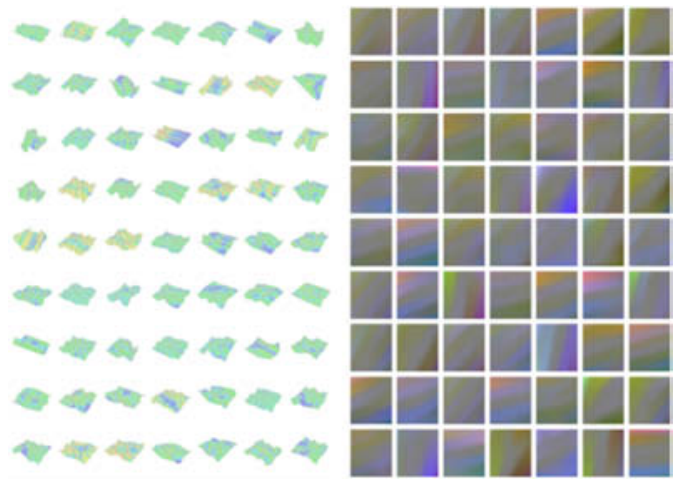

Figure 9. Simulation Results and Output Displacement Images: the $\mathrm{x}, \mathrm{y}, \mathrm{z}$ displacement values were into rgb.

vertices displacements were mapped into rgb values (Figure 9). The image pairs were then divided equally into a training and a testing set. After the model was trained, the cutout pattern from the testing set was simulated to evaluate the accuracy of the model. Figures 11 and 12 show that this model had a higher RMSE than the first model as the required input deformation is more complex. The average RMSE for all samples was 0.42 . However, Figure 10 shows that the predicted model is visibly similar to the desired mesh and was produced in a fraction of a second compared to running a standard FEM simulation. This means that the method could be more suited to applications that do not require high precision. To increase the accuracy of the method, given a desired membrane deformation and after the cutout pattern is generated from the ML model, another optimization step could be done to reach the desired deformation with a lower RMSE.

\section{CONCLUSIONS}

This research proposed a new workflow for the design of complex deployable three-dimensional structures using machine learning and nonlinear FEA simulation. Machine learning presents a unique opportunity in

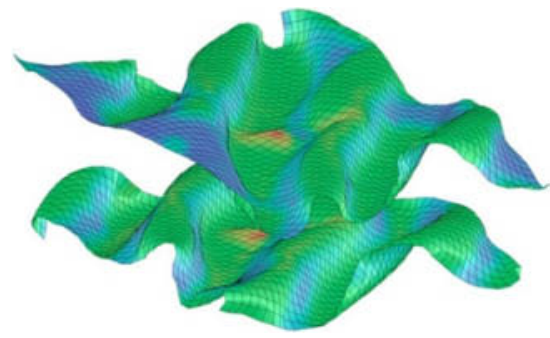

Figure 10. Example of Predicted vs Desired Mesh Deformation.

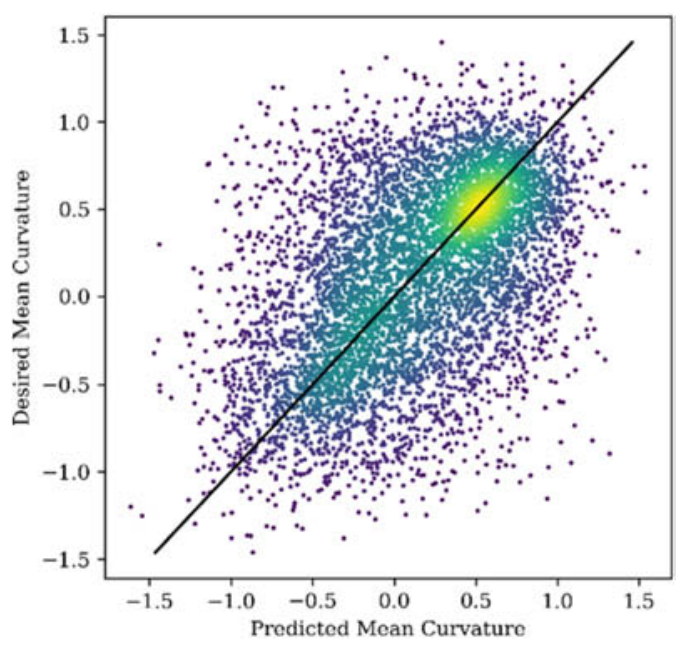

Figure 11. Predicted vs Desired Mean Curvature for all the points in all the samples for Model 2.

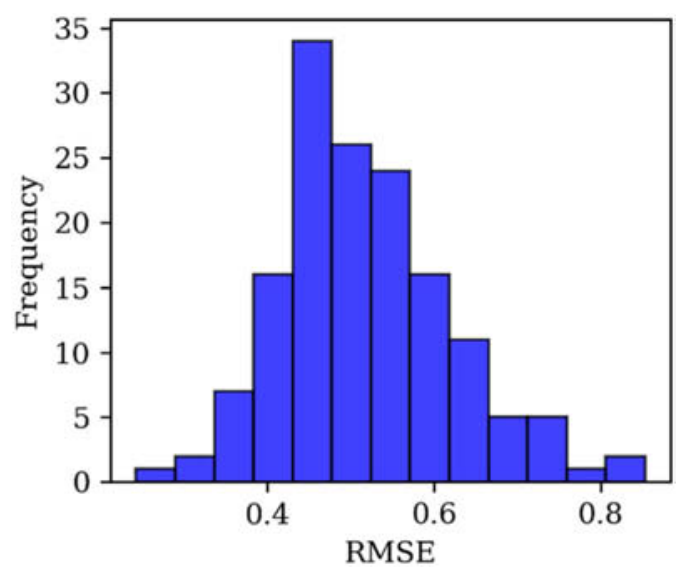

Figure 12. Probability Density Function for Root Mean Squared Error for Model 2.

terms of the ability to solve correlations that were believed to be unsolvable using any other method. The workflow also offers a distinctive advantage compared to other evolutionary and optimization techniques 
since, once the model is trained, the output is instantaneous and within the acceptable level accuracy; one can get real time results for a desired input. The proposed workflow offers a fast and systematic way for the design and exploration of thermally actuated structures. In future research, this method will be extended to model other actuation techniques, shapes, systems (origami and kirigami), as well as any other three-dimensional geometries.

\section{REFERENCES}

Abadi, M., Barham, P., Chen, J., Chen, Z., Davis, A., Dean, J. \& Zheng, X. (2016). TensorFlow: A System for Largescale Machine Learning. Proc. 12th USENIX Conference on Operating Systems Design and Implementation (pp. 265-283). Berkeley, CA, USA: USENIX Association. Retrieved from http://dl.acm.org/citation.cfm? $\mathrm{id}=3026877.3026899$

Addington, M., \& Schodek, D. (2005). Smart Materials and New Technologies - For architecture and design professions. Elsevier, 1-254.

Bishop, C. M. Pattern Recognition and Machine Learning (Springer, 2006)

Boatti, E., Vasios, N., \& Bertoldi, K. (2017). Origami Metamaterials for Tunable Thermal Expansion. Advanced Materials, 1700360, 1700360. https://doi.org/10.1002/ adma.201700360

Boatti, E., Vasios, N., \& Bertoldi, K. (2017). Origami Metamaterials for Tunable Thermal Expansion. Advanced Materials, 1700360, 1700360. https://doi.org/10.1002/ adma.201700360

Carmody, J., Selkowitz, S., Lee, eleanor s., Arashteh, D., \& Willmert, T. (2004). Window System for HighPerformance Buildings, WW Norton \& Company.

Connolly, F., Walsh, C. J., \& Bertoldi, K. (2016). Automatic design of fiber-reinforced soft actuators for trajectory matching. Proceedings of the National Academy of Sciences, 114(1), 201615140. https://doi.org/10.1073/pnas. 1615140114

Deng, B., Raney, J. R., Tournat, V., \& Bertoldi, K. (2017). Elastic Vector Solitons in Soft Architected Materials. Physical Review Letters, 118(20), 204102. https://doi. org/10.1103/PhysRevLett.118.204102

Deng, B., Raney, J. R., Tournat, V., \& Bertoldi, K. (2017). Elastic Vector Solitons in Soft Architected Materials. Physical Review Letters, 118(20), 204102. https://doi. org/10.1103/PhysRevLett.118.204102

Deng, B., Raney, J. R., Tournat, V., \& Bertoldi, K. (2017). Elastic Vector Solitons in Soft Architected Materials. Physical Review Letters, 118(20), 204102. https://doi. org/10.1103/PhysRevLett.118.204102

Javid, F., Smith-Roberge, E., Innes, M. C., Shanian, A., Weaver, J. C., \& Bertoldi, K. (2015). Dimpled elastic sheets: a new class of non-porous negative Poisson's ratio materials. Scientific Reports, 5(1), 18373. https://doi. org/10.1038/srep 18373

H.T. Maune, S.-p. Han, R.D. Barish, M. Bockrath, W.A. Goddard III, P.W. Rothemund \& E. Winfree, Selfassembly of carbon nanotubes into two-dimensional geometries using DNA origami templates, Nature Nanotechnol. 5 (1) (2010) 61-66

Isola, P., Zhu, J.-Y., Zhou, T., \& Efros, A. A. (2016). Image-to-Image Translation with Conditional Adversarial Networks. arXiv, 16. https://doi.org/arXiv:1611.07004
Jordan, M. I. \& Mitchell, T. M. Machine learning: trends, perspectives, and prospects. Science 349, 255-260 (2015)

Khoo, Z. X., Teoh, J. E. M., Liu, Y., Chua, C. K., Yang, S., An, J. \& Yeong, W. Y. (2015). 3D printing of smart materials: A review on recent progresses in 4D printing. Virtual and Physical Prototyping, 10(3), 103-122. https://doi.org/10.1080/17452759.2015.1097054

Kolarevic, B. \& Malkawi, A. Performative Architecture: Beyond Instrumentality; Routledge: London, UK, 2005.

Liu, J., \& Bertoldi, K. (2015). Bloch wave approach for the analysis of sequential bifurcations in bilayer structures. Proceedings of the Royal Society A: Mathematical, Physical and Engineering Science, 471(2182), 20150493. https://doi.org/10.1098/rspa.2015.0493

Liu, J., Gu, T., Shan, S., Kang, S. H., Weaver, J. C., \& Bertoldi, K. (2016). Harnessing Buckling to Design Architected Materials that Exhibit Effective Negative Swelling. Advanced Materials (Deerfield Beach, Fla.), 28(31), 6619-24. https://doi.org/10.1002/adma.201600812

Majidi, C. (2014). Soft Robotics: A Perspective-Current Trends and Prospects for the Future. Soft Robotics, 1(1), 5-11. https://doi.org/10.1089/soro.2013.0001

Mousanezhad, D., Babaee, S., Ebrahimi, H., Ghosh, R., Hamouda, A. S., Bertoldi, K., \& Vaziri, A. (2015). Hierarchical honeycomb auxetic metamaterials. Scientific Reports, 5(1), 18306. https://doi.org/10.1038/srep18306

Peraza-Hernandez, E. A., Hartl, D. J., Malak, R. J., \& Lagoudas, D. C. (2014). Origami-inspired active structures: A synthesis and review. Smart Materials and Structures, 23(9). https://doi.org/10.1088/0964-1726/23/ 9/094001

Rafsanjani, A., \& Bertoldi, K. (2017). Buckling-Induced Kirigami. Physical Review Letters, 118(8). https://doi.org/ 10.1103/PhysRevLett.118.084301

Ramprasad, R., Batra, R., Pilania, G., Mannodi-Kanakkithodi, A., \& Kim, C. (2017). Machine Learning and Materials Informatics: Recent Applications and Prospects. Npj Computational Materials, (November). https://doi.org/10. 1038/s41524-017-0056-5

Shan, S., Kang, S. H., Zhao, Z., Fang, L., \& Bertoldi, K. (2015). Design of planar isotropic negative Poisson's ratio structures. Extreme Mechanics Letters, 4, 96-102. https://doi.org/10.1016/j.eml.2015.05.002

Szokolay, S. Introduction to Architectural Science: The Basis of Sustainable Design; Routledge: London, UK, 2008

Tolley, M. T., Felton, S. M., Miyashita, S., Aukes, D., Rus, D., \& Wood, R. J. (2014). Self-folding origami: shape memory composites activated by uniform heating. Smart Materials and Structures, 23(9), 94006. https://doi.org/ 10.1088/0964-1726/23/9/094006

Wang, Z., Polygerinos, P., Overvelde, J. T. B., Galloway, K. C., Bertoldi, K., \& Walsh, C. J. (2017). Interaction Forces of Soft Fiber Reinforced Bending Actuators. IEEE/ASME Transactions on Mechatronics, 22(2), 717727. https://doi.org/10.1109/TMECH.2016.2638468

Xue, R., Li, R., Du, Z., Zhang, W., Zhu, Y., Sun, Z., \& Guo, X. (2017). Kirigami pattern design of mechanically driven formation of complex 3D structures through topology optimization. Extreme Mechanics Letters, 15, 139-144. https://doi.org/10.1016/j.eml.2017.03.004

Y. Zhang, Z. Yan, K. Nan, D. Xiao, Y. Liu, H. Luan, H. Fu, X. Wang, Q. Yang, J. Wang, et al., A mechanically driven form of Kirigami as a route to 3D mesostructures in micro/nanomembranes, Proc. Natl. Acad. Sci. USA 112 (38) (2015) 11757-11764. 